JURNAL

ACTE DE LANGAGE DE SUGGESTION DANS LE FILM «INTOUCHABLES "

Disusun dan Diajukan oleh:

RENTA LUBIS

NIM : 2123131036

Telah Diverifikasi dan Dinyatakan Memenuhi Syarat untuk Diunggah pada Jurnal Online

Medan, November 2017

Menyetujui,

Pembimbing Skripsi I,

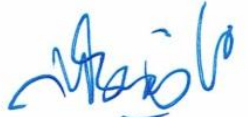

Dr. Marice, M.Hum

NIP. 196311201992032003
Pembimbing Skripsi II,

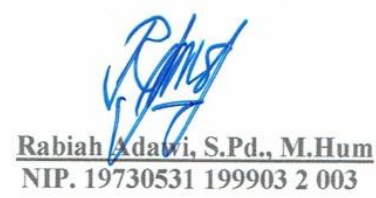

Editor Jurnal

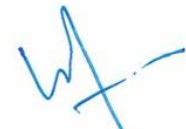

Nurilam Harianja, S.Rd., M.Hum

NIP. 197701212003122004 


\title{
ACTE DE LANGAGE DE SUGGESTION DANS LE FILM «INTOUCHABLES »
}

\author{
Renta Lubis \\ Dr. Marice, M.Hum \\ Rabiah Adawi, S.Pd, M.Hum \\ Département des Langues Étrangères \\ Faculté des Lettres et des Arts \\ Université de Medan \\ Mèl : rentalubis55@gmail.com \\ RÉSUMÉ
}

\begin{abstract}
Renta Lubis, NIM. 2123131036. "Acte de Langage de Suggestion dans le Film "Intouchables "”. Mémoire. Section Française du Département de Langue Étrangère, Faculté des lettres et des arts. Université de Medan. 2017.
\end{abstract}

Le but de cette recherche est de trouver quelles sont les expressions de suggestion utilisées et les modes utilisées dans le film Intouchables. Les catégories de ce type de l'acte de langage viennent de classification proposée par Martinez-Flor (2005) qui regroupent l'acte de langage de suggestion en trois : directe, conventionnelle, et indirecte. La méthode de la recherche est la méthode qualitative. La méthode qualitative est une donnée qui est désignée par les mots ou bien les phrases qui sont séparées selon la catégorie précisée pour obtenir la conclusion.

Le résultat de la recherche indique que les expressions de l'acte de suggestion dans le film Intouchables sont variées. Ce sont, les expressions de performatif (1), les expressions d'impératif (9), les expressions d'impératif négatif (1), les expressions de possibilité (1), les expressions de devoir (3), les expressions de besoin (2). On trouve quelques modes d'emploi pour exprimer les suggestions, ce sont les modes l'indicatif présent et l'impératif présent. Selon les données qui sont trouvées les expression de suggestion qui sont plus dominantes dans le film Intouchables est les expressions de suggestion directe avec le type d'impératif. Il signifie que le locuteur exprime toujours ses suggestions directement, alors que l'interlocuteur les comprend facilement.

Mots-clés : Acte de langage, expressions de suggestions, film Intouchables.

\begin{abstract}
ABSTRAK
Renta Lubis, NIM. 2123131036. "Acte de Langage de Suggestion dans le Film "Intouchables "”. Mémoire. Section Française du Département de Langue Étrangère, Faculté des lettres et des arts. Université de Medan. 2017.

Adapun yang menjadi tujuan pada penelitian ini yaitu untuk menemukan ekspresi saran apa saja yang digunakan dan modus kalimat yang digunakan dalam film « Intouchables». Pengelompokan kategori dan jenis tindak tutur ini berasal dari
\end{abstract}


klasifikasi yang disusun oleh Martinez-Flor yang mengklasifikasikan tindak tutur ke dalam tiga bagian yaitu, langsung, conventionel dan tidak langsung. Metode penelitian yang digunakan adalah metode kualitatif. Metode kualitatif adalah metode yang digambarkan dengan kata-kata atau kalimat dan dapat dipisahpisahkan menurut kategori untuk memperoleh kesimpulan.

Hasil penelitian menunjukkan bahwa tindak tutur saran dalam film Intouchables beragam. Hasil Ekspresi performatif adalah (1), Ekspresi perintah (9), Ekspresi perintah negatif(1), Ekspresi kemungkinan (1), Ekspresi harus (3), Ekspresi kebutuhan(2). Dalam penelitian ini ditemukan penggunaan beberapa Modus untuk memberikan saran, yaitu modus Indicatif Présent, Imperatif. Berdasarkan data yang diperoleh, ekspresi saran yang paling sering muncul digunakan penutur dalam film Intouchables adalah ekspresi saran secara langsung dengan tipe perintah.Hal tersebut menunjukkan bahwa penutur cenderung mengekspresikan sarannya dengan cara memberikan saran secara langsung, agar lawan tutur mudah memahami maksud si penutur.

\section{Kata kunci : Tindak tutur, ekspresi saran, film Intouchables.}

\section{INTRODUCTION}

Les gens utilisent la langue pour communiquer dans la vie quotidienne. Une langue est un instrument de communication qui est utilisée par chaque personne pour exprimer les idées, les sentiments et les pensées. Donc, la langue tient un rôle très important dans la vie des humains. dans tous les processus de communication, il c'est ce qu'on appelle un «événement de parole» et «actes de parole» dans une situation de parole. Il y a beaucoup de types de l'acte de langage : acte de langage de requête, de remerciement, d'excuse, de compliment, de salutation, d'appréciation, de suggestion, etc. Les suggestions sont utilisées dans la vie quotidienne, parce que les gens utilisent les suggestions pour aider les interlocuteurs a faire quelque chose ayant une valeur qui donne pendant le cours des avantages. Les français utilisent toujours la suggestion par exemple quand ils donnent la suggestion à leur amis, la famille, etc. Il existe des exemples l'acte de langage de suggestion que les français utilisent souvent dans leur phrase, comme: Je suggère que vous me donniez une réponse maintenant. Ils se trouve plusieurs variations des expressions des suggestions. Selon Martinez-Flor (2005:168), les expressions des suggestions sont variées. Il classifie les expressions de suggestions en trois types, ce sont la suggestion directe, conventionnelle, et indirecte. Par conséquent, cette recherche est très importante à faire pour informer au lecteur, en particulier les français sur la variation de acte de langage de suggestion. 


\section{RECOURS AUX THÉORIES}

\section{Pragmatique}

La pragmatique est un domaine de la linguistique assez récent. Selon Lech (1983 : 1) la pragmatique n'était pas encore une branche reconnue, si l'on la compare à la phonologie, à la morphologie ou à la syntaxe. Pour sa part, la pragmatique contient des aspects que les chercheurs ne peuvent pas placer dans les domaines établis de la linguistique. Pragmatique est à l'origine d'une partie de la philosophie du langage. Elle a contribué à l'élaboration conceptuelle des approches communicatives.

\section{Les Actes des Langages}

La langue constitute un moyen d'agir parmi d'autres, 'dire, c'est faire'. Selon Yule (1996 : 48 ), l'acte de langage est un acte qui se fait par la parole. Yule classifie les actes de langage en cinq catégories : a. Les actes "directifs", le locuteur a demandé l'auditeur de faire quelque chose ( ordre, requétes, suggestion, etc.), b. Les actes "déclaratifs", c. Les actes "représentatifs", d. Les actes "expressifs" (remerciements, félicitations), e. Les actes "commissives" (promesse).

\section{L'acte de Langage Directif}

L'acte illocutoire directif est une tentative de faire quelque chose à l'auditeur. Elle révèle ce qui est souhaite par l'orateur. Sa fonction est de conseiller, demander, supplier, interdit, insister, suggérer, inviter, commander, permettre, recommander, montrer.

Yule (1996 :55) explique complètement que :

Directive are those kinds of specch acts that speakers use to get someone else to do something. They express what the speaker wants. They are commands, orders, requests, suggestions.

Cela veut dire que l'acte illocutoire directifest les types d'actes de langage où les locuteurs le demandent aux interlocuteurs de faire quelque chose. Il explique ce que le locuteur veut. Il s'agit les ordres, les requétes, et les suggestions.

\section{Suggestion}

Selon Banerjee (1988), la suggestion est une parole du locuteur pour influencer l'interlocuteur pour faire quelque chose de l'avantage. Martinez-Flor présente trois type des suggestions : 1) Suggestion Directe .2) Suggestion Conventionelle. 3) Suggestion Indirecte. 
Tableau les Types des Suggestions Directes

\begin{tabular}{|c|c|c|}
\hline Type & Classifications & Exemple \\
\hline \multirow[t]{3}{*}{ Directe } & Performatif & $\begin{array}{l}\text { Je pense que vous devriez tenter une autre } \\
\text { voie } \\
\text { Je vous conseille de prendre vos billets à } \\
\text { l'avance } \\
\text { Je vous recommande de bien l'accueillir } \\
\text { Je suggère que vous me donniez une réponse } \\
\text { maintenant }\end{array}$ \\
\hline & Impératif & Parlez plus haut, s'il vous plait \\
\hline & Impératif négatif & Je veux pas que tu joues avec la boîte de sucre \\
\hline
\end{tabular}

Tableau les Types des Suggestions Conventionnelle

\begin{tabular}{|c|l|l|}
\hline Type & Classifications & \multicolumn{1}{c|}{ Exemple } \\
\hline \multirow{5}{*}{ Conventionnelle } & Interrogatif & $\begin{array}{l}\text { Pourquoi ne pas aller à la patinoire à la } \\
\text { place ? } \\
\text { Ça te dirait une partie de golf demain } \\
\text { matin ? } \\
\text { Et si on allait faire les boutiques avec } \\
\text { lui, pour une fois ? }\end{array}$ \\
\cline { 3 - 4 } & & Vous pourriez l'écouter! \\
\cline { 2 - 3 } & Possibilité & Tu devrais remonter ce bouton-là \\
\cline { 2 - 3 } & Devoir & Tu as besoin de repos \\
\cline { 2 - 3 } & Besoin & $\begin{array}{l}\text { Si j'avais assez d'argent, je voudrais } \\
\text { acheter une voiture }\end{array}$ \\
\cline { 2 - 3 } & Conditionnel \\
\hline
\end{tabular}

Tableau le Type de Suggestion Indirecte

\begin{tabular}{|c|c|c|}
\hline Type & Classification & Exemple \\
\hline Indirecte & Impersonnel & C'est mieux que tu finis tes devoirs à la maison. \\
\hline
\end{tabular}

\section{MÉTHODOLOGIE DE LA RECHERCHE}

La méthode de recherche est très importante dans une recherche afin que le résultat obtenu soit satisfaisant. Avec la méthode de recherche correcte, les problèmes apparus dans la recherche peuvent être résous et le chercheur peut trouver les vérités, si bien que dans l'analyse de données, il peut être bien dirigé. Le processus de recherche qui est orientée va produire l'information et l'analyse de données. Cette information peut être acceptée naturellement par tout le monde.

Pour analyser les données dans cette recherche, on utilise la méthode qualitative. Selon Moleong ( 2001 : 178) «metedologi kualitatif sebagai prosedur 
penelitian yang menghasilkan data deskriptif berupa kata-kata tertulis atau lisan dari orang-orang dan perilaku yang dapat diambil».

La technique du collecte de données est la metode simak. Selon Sudaryanto (1993 :192) la metode simak est une méthode qui est faite en regardant ou observant l'utilisation de langue. C'est à dire la metode simak est une méthode d'étude du document.

Les procédures d'analyse de données dans cette recherche se composent de :

1. Voir le film «Intouchables » et le tourner plusieurs fois.

2. Faire la transcription du dialogue concernant les suggestions.

3. Identifier les expressions de suggestions qui se trouve dans le film Intouchables.

4. Identifier et analyser des modes utilisés dans les dialogues.

5. Grouper les catégories de l'acte de langage de suggestion.

6. Faire le tableau de l'acte de langage de suggestion qui se trouve dans le film Intouchables.

7. Analyser le mode de l'acte des expressions de suggestion dans le film utilisé par les français.

8. Conclure le résultat de la recherche.

\section{RÉSULTAT DE LA RECHERCHE}

Après avoir analysé les données dans le film Intouchables qui dure pendant deux heures et il consiste 1500 dialogues, on trouve finalement 17 expressions de suggestions. On peut les classifier comme: 1. acte de langage de suggestion directe qui atteint 13 expressions. Ce sont : le performatif qui atteint 1 expression, l'impératif atteint 9 expressions et 1 ' impératif négatif atteint 1 expression. 2 . Actes de langage de suggestion conventionnelle atteint 7 expressions. Ce sont: la possibilité qui atteint 1 expression, le devoir atteint 3 expressions et le besoin atteint 2 expressions.

On trouve aussi deux modes d'emploi pour exprimer les suggestions dans le film Intouchables, ce sontl'indicatif présent et l'impératif. Donc, pour que le résultat de la recherche soit bien à voir, on met le résultat de la recherche dans le tableau suivant

\section{Tableau 4.1}

Tableau Récapitulatif de la Recherche des Actes de Langage Suggestions Dans le Film Intouchables

\begin{tabular}{|c|l|c|}
\hline \multirow{2}{*}{ NO } & \multicolumn{1}{|c|}{ Type de L'acte de Langage de Suggestion } & Fréquence \\
\hline 1 & Directe & 1 \\
\hline \multirow{2}{*}{} & Performatif & 9 \\
\cline { 2 - 3 } & Impératif & 1 \\
\cline { 2 - 3 } & Impératif Négatif \\
\hline
\end{tabular}




\begin{tabular}{|c|l|c|}
\hline 2 & Conventionnelle & \\
\hline & Possibilité & 1 \\
\hline \multirow{2}{*}{ TOTAL } & Devoir & 3 \\
\cline { 2 - 3 } & Besoin & 2 \\
\hline \multirow{2}{*}{ TOL } & 17 \\
\hline
\end{tabular}

\section{Les Types d'Acte de Langage de Suggestion Directe}

Le type d'acte de langage de suggestion est la suggestion directe que le locuteur explique clairement ce qu'il a dit. Dans le film Intouchables, on trouve les expressions de suggestions directes, ce sont les expressions de performatif (1), impératif (10) et impératif négatif (1).

Voici les examples :

\section{a. Détendez-vous !}

Cette parole "détendez-vous", prononcée par Driss à Philippe, c'est une expression de la suggestion d'impératif (exprimer principalement l'ordrepeut aussi exprimer la suggestion) parce qu'il utilise la phrase impérative. Dans cette situation, Driss et Philippe sont en train d'etrê controlés à l'hôpital. Philippe semble inquiet, Driss lui parle en donnant la suggestion pour se détendre afin que le docteur puisse bien éxaminer sa santé.

\section{b. Je pense que c'est d'aider l'autre.}

Cette parole prononcée par Magalie à Driss pendant l'entretien de chercher un nouveau gardien. Magalie questionne la motivation de Driss. Et puis, il réponse que ses motivations sont pour l'argent et l'humain. Donc, Magalie donne une suggestion en disant "Je pense, c'est d'aider l'autre" .On appelle expression de la suggestion de performatif( la parole qui a été déjà accomplie par le locuteur) parcequ'il utilise le verbe "penser" et cette suggestion a été faite ence moment- là.

\section{Les Types d'Acte de Langage de Suggestion Conventionnelle}

Le deuxième groupe de la suggestion, c'est la conventionnelle. Elle est utilisée à faire une suggestion, il est possible que le locuteur peut comprendre le but de la suggestion. Dans le film Intouchables, on trouve plusieurs expressions de suggestions conventionnelle ce sont possibilité (1 expression), devoir (3 expressions), besoin ( 2 expressions).

\section{a. Tu dois garder les articulations en bon état}

Cette parole "tu dois garder les articulations en bon état", prononcée par Marcelle à Philippe, c'est une expression de la suggestion de devoir parce qu'il utilise le verbe dois. Marcelle donne la suggestion à Philippe pour garder son état en faisant 
du sport. On trouve qu'il y a deux modes que l'on utilise pour exprimer les suggestions, ce sont les modes : indicatif présent et l'impératif.

b. Je vous pensepouvoir supporter des contraintes, des horaires. .

Cette parole "Je vous pense pouvoir supporterdes contraintes, des horaires”, prononcée par Driss à Philippe, c'est une expression de la suggestion de possibilité parcequ'ilutilise le verbe "pourrait". Dans cette situation, Driss à Philippe se mettent d'accord pour aller à cinéma. Alors, Driss donne la suggestion à Philippe pour manager les horaires.

\section{CONCLUSION}

En se basant sur le résultat de la recherche, on peut tirer la conclusion suivante : Les expressions de suggestion utilisées dans le film Intouchables sont variées, ce sont : 1 . Expression de Suggestion Directe : Performatif (1 expression), Impératif (9 expressions ), Impératif Négatif( 1 expression ) 2. Expression de Suggestion Conventionnelle : Possibilité (1 expression), Devoir (3 expresiions), Besoin ( 2 expressions)

Selon les données trouvées, l'expression de suggestion qui est plus dominante dans le film Intouchables est l'expression de suggestion directe avec le type d'impératif. Il signifie que le locuteur exprime toujours ses suggestions directement à l'interlocuteur pour que ce-dernier les comprennent facilement de ce que le locuteur lui parle, alors l'interlocuteur doit faire une action.

On trouve qu'il y a deux modes que l'on utilise pour exprimer les suggestions, ce sont les modes : indicatif présent et l'impératif. L'utilisation de l'indicatif présent à l'impératif se trouve souvent dans ce film parce qu'il parle de la vie quotidienne racontant les activités et les situations des sens quand ils se communiquent.

\section{BIBLIOGRAPHIE}

Austin J. L. 1962. How To Do Things With Words. Clarendon Press: Oxford.

Banerjee, J., \& Carrell, P.L. (1988). Tuck in your shirt, you squid: Suggestions in ESL. Language Learning.

Cuq, Jean-Pierre.2003. Dictionnaire de Didactique du Français. Paris. CLE International.

Jean M. R et Isabelle C.2009. Précis de Grammaire. Paris. CLE International.

Levinson, Stephen C. 1983. Pragmatics, United Kingdom: Cambridge.

Lech, G.N. 1983. Principales Of Pragmatics. Longman : Londres et New York

Maris Juliana, Rut, 2015. Acte de Langange d'interdiction dans Le Film Le Petit Nicolas, Medan : Universitas Negeri Medan. 
Margono, 2005. Penelitian Kualitatif sebagai Dasar Analisis. Bandung : Cipta Jaya.

Martínez-Flor, Alicia (2005): A Theoretical Review of the Speech Act of Suggesting:Towards a Taxonomy for its Use in FLT1. Doctoral Dissertation. Castellón: Universitat Jaume I.

Meilala, Memoire. 2015. Analyse de l'acte de langage exprimant de l'opinion dans le film Ratatouille. Medan : Universitas Negeri Medan.

Moleong. 2001. MetodePenelitianKualitatif. Semarang : BuanaJaya.Nadar .F. R.2009. Pragmatik dan penelitian Pragmatik. Yogyakarta. Graha Ilmu.

Nababan, P.W.J. 1984. Ilmu Pragmatik (Teori dan penerapannya). Jakarta: Departemen Pendidikan Indonesia.

Nadar. F. X. 2009. Pragmatik Dan Penelitian Pragmatik. Yogyakarta. Graha Ilmu.

Rintell, Ellen (1979): "Getting your speech act together: The pragmatic ability of second language leamers". Working Papers on Bilingualism.

Searle, J. 1975. Speech Acts: An Essay in the Philosophie of Language. Cambridge, England. Cambridge University Press.

Sitepu, 2014, Acte de Langange de Requête Des Etudiants du Quatrième Semestre de La Section Française de L'Université de Medan L'année Universitaire 2013/2014, Medan : Universitas Negeri Medan.

Tarigan, Agus. 2017. Variation de Pronom ON Dans Le Roman 99 Francs. Medan: Universitas Negeri Medan.

Yule, George. 1996. Pragmatics. New York: Oxford University Press.

http://www.anglaisfacile.com/exercices/exercice-anglais2/exerciceanglais 28230.php, consulté le 26 juin, $10.00 \mathrm{WIB}$

http://fr.wikipedia.org/wiki/Acte de langage, consulté le 12 juin 2016, 14.00 WIB

https://fr.wikipedia.org/wiki/Intouchables_(film), consulté le 23 août 2016, 09.15 WIB 